\title{
Modification of Diazoquinone-Novolac Photoresist Films by the Implantation of Antimony Ions
}

\author{
S. D. Brinkevich ${ }^{a}$, D. I. Brinkevich ${ }^{a, *}$, and V. S. Prosolovich ${ }^{a, * *}$ \\ ${ }^{a}$ Belarusian State University, Minsk, 220013 Belarus \\ *e-mail: Brinkevich@bsu.by \\ **e-mail:prosolovich@bsu.by
}

Received May 20, 2020; revised May 20, 2020; accepted May 26, 2020

\begin{abstract}
In this paper, we study the radiation-induced processes occurring during the implantation of antimony ions into films of the positive diazoquinone-novolac (DQN) FP9120 photoresist (PR) on silicon by the Fourier-transform infrared (FTIR) spectroscopy of the frustrated total internal reflection (TIR). Ion implantation (II) is found to lead to the appearance in the frustrated TIR spectrum of a band at $2331 \mathrm{~cm}^{-1}$ caused by the $\mathrm{O}=\mathrm{C}=\mathrm{O}$ stretching vibrations. The violation of adhesion at the $\mathrm{PR} /$ silicon interface manifests itself in the appearance of a $610 \mathrm{~cm}^{-1}$ band related to the absorption of the Si lattice. The formation of new $\mathrm{C}-\mathrm{O}-\mathrm{C}$ bonds because of the ether cross links of ketene with the $\mathrm{OH}$ group of novolac resin is found.
\end{abstract}

Keywords: frustrated total internal reflection spectrum, diazoquinone-novolac photoresist, implantation, adhesion, silicon

DOI: $10.1134 / \mathrm{S} 1063739720060025$

\section{INTRODUCTION}

At present, lithography is one of the main processes in the general production cycle of a wide class of semiconductor devices and integrated circuits. Diazoquinone-novolac (DQN) resists are widely used as masks in submicron and nanolithography [1-4]. One of the main methods for the formation of doped regions in modern electronics is ion implantation (II). It allows accurately controlling the dopant concentration and is characterized by the versatility and flexibility of the process. The interaction of DQN resists with ultraviolet, X-ray, and visible radiation has been studied in sufficient detail, while the processes induced by ion irradiation remain poorly understood, despite the fact that they can have a significant effect on the quality of the created devices [4-6].

During the II of polymers, radiation-induced processes were previously shown to occur both in the region of the ion path and outside it [5, 7-9]. For example, the radiation hardening of DQN resist films behind the layer of antimony ions implantation was discovered in [5]. However, the mechanism of the radiation-induced processes responsible for the change in the physicomechanical properties of DQN resists behind the layer of II was not established.

For the thin film studies, the FTIR spectroscopy of the frustrated total internal reflection (TIR) that allows obtaining qualitative and quantitative information on the composition and structure of complex organic compounds and their mixtures in the solid aggregation state is widely used [10]. Note that the frustrated TIR method was not used earlier to study DQN photoresist (PR) films on the surface of monocrystalline silicon wafers. In this study, we used the FTIR spectrometry of the frustrated TIR in order to establish the mechanisms of the radiation-induced modification of the physicomechanical properties of DQN PR films upon implantation of antimony ions.

\section{EXPERIMENTAL}

A positive FP9120 PR is a composite of light-sensitive $o$-naphthoquinone-diazide and phenol-formaldehyde resin. PR films with a thickness of $1.8 \mu \mathrm{m}$ were deposited on the surface of $p$-type silicon wafers $(\rho=$ $10 \Omega \mathrm{cm}$ ) with the (111) orientation by the centrifugation method at the angular velocity of $V=2900 \mathrm{rpm}$ [11]. Before the formation of the PR film, the silicon wafers were subjected to the standard cycle of surface cleaning in organic and inorganic solvents and washing in deionized water. After applying the PR to the operating side of the plate, it was dried for $50-55 \mathrm{~min}$ at $88^{\circ} \mathrm{C}$. The thickness of the PR films was monitored using a Dectak profilometer.

Implantation with $\mathrm{Sb}^{+}$antimony ions was carried out with an energy of $60 \mathrm{keV}$ in the dose range of $\Phi=1 \times 10^{15}-5 \times 10^{16} \mathrm{~cm}^{-2}$ at an ion current density of $j=4 \mu \mathrm{A} / \mathrm{cm}^{2}$ in a residual vacuum not worse than $10^{-5} \mathrm{~mm} \mathrm{Hg}$ using the Vezuvii-6 ion-beam accelerator. The frustrated-TIR spectra of the PR-silicon struc- 